\title{
Discurso en acto de inauguración XVI JUDC UNAN FAREM-Estelí
}

\section{Speech at opening ceremony XVI JUDC UNAN FAREM- Estelí}

\author{
Dr. Manuel Enrique Pedroza \\ Director de investigación UNAN-Managua
}

\begin{abstract}
Septiembre mes de la patria, mes de heroicos hijos de la patria que nos heredaron el presente de paz y estabilidad que hoy disfruta nuestra juventud
\end{abstract}

Saludos a las Cras. y Cros. presentes

En principio deseo saludarlos y felicitarlos por este hermoso día 18 de septiembre en que nos encontramos reunidos para celebrar la XVI JUDC 2013 de FAREM Estelí, UNAN-Managua. Este heroico mes de septiembre, que está lleno de triunfos y de glorias populares a lo largo y ancho de nuestra historia, entre ellos: El ejemplo del General. José Dolores Estrada, en La Batalla de San Jacinto el 14 de septiembre de 1856 y es también celebrar la firma de nuestra Independencia del Colonialismo español, el 15 de septiembre de 1821.

Septiembre, mes glorioso que está lleno de heroísmo. Septiembre heroico, es recordar el ejemplo inmortal de Salvador Allende en Chile que hace 40 años, el 11 de sept del 1973, cae abatido por las balas asesinas del dictador chileno Augusto Pinochet. Septiembre heroico, es recordar el martirologio de Victor Jara, cantautor Chileno que hace 40 años, el 16 de sept del 1973, cae asesinado por la dictadura de Pinochet.

Septiembre heroico, es recordar el ejemplo inmortal de Leonel Rugama, que sembró la semilla de la revolución en los años difíciles de la siembra, cuando la cosecha aún era solo una esperanza. Septiembre heroico, es recordar el ejemplo inmortal del Cmdte. Ricardo Morales Avilés, que un día como hoy 18 de sept, pero hace 40 años, cae asesinado por las balas asesinas de la dictadura Somocista, y junto con él en Nandaime caen Oscar Turcios, Jonathan González y Juan José Quezada.

En Septiembre heroico, también celebramos la caída heroica de Bernardino López Ochoa, un 3 de Sept y por eso, es el 3 de sept la fecha en que en Nicaragua celebramos El día del Campesino Nicaragüense.

El 21 de Septiembre, también celebramos la caída heroica de Rigoberto López Pérez, luego de ajusticiar al fundador de la dictadura somocista. 
Nuestra historia patria, se ha escrito con éxitos y reveses. La lección aprendida que nos enseña nuestra historia patria, es que lo importante es mirar al futuro, con identidad de patria, con compromiso social revolucionario. Septiembre heroico, es recordar el ejemplo inmortal de nuestros héroes y mártires que nos heredaron el presente luminoso de paz y estabilidad del que hoy disfruta nuestra juventud.

\section{La XXXII Jornada Universitaria de Desarrollo Científico, JUDC-2013}

\section{EI Contexto para celebrar la XVI JUDC 2013 de FAREM-Estelí, UNAN-Managua}

Con el triunfo de nuestra revolución Popular Sandinista, el 19 de Julio de 1979, se implementaron una serie de transformaciones estratégicas en la Sistema de Educación Superior de Nicaragua. Una de ellas fue la creación del CNES, (Consejo Nacional de Educación Superior) que derivo años después en el actual (Consejo Nacional de Universidades). El CNES orientó la creación de las DIP (Direcciones de Investigación y Posgrado), que derivaron años después en las actuales Vicerrectorías de Investigación y Posgrado o simplemente DIEP.

Hace treinta y dos año se orientó a través de las DIP, la realización de las Jornadas Universitarias de Desarrollo Científico, JUDC, como un espacio de participación estudiantil para el intercambio académico, el compartir conocimientos, habilidades y destrezas de los jóvenes estudiantes universitarios, que permitiera mostrar los avances en materia de investigación científica de cada universidad.

En nuestra "alma mater" la UNAN-Managua, se organiza la XXXII JUDC 2012, con el lema "Por la excelencia académica, investigando e innovando". A la par del XVIII Congreso Científico de UNAN-Managua, la XXXII JUDC 2012, se viene organizando desde inicios del mes de mayo de 2013, para promover entre otros aspectos, el rol de la Investigación Científica en el trabajo universitario y su articulación con la Docencia y la Extensión Universitaria.

Organizada y dirigida por la Comisión de Investigación de FAREM Estelí, la XVI JUDC 2013, está desarrollando la Investigación Formativa en la juventud heroica de Estelí, incentivando y organizando la participación estudiantil, en las actividades de investigación científica con el apoyo de los docentes, en su calidad de tutores o al final como miembros de tribunal examinador de la XVI JUDC, quienes orientan a una mejor participación desde el aula de clase, promoviendo a los estudiantes a presentar desde un ensayo, un protocolo, un proyecto de desarrollo, un diseño de página web, una aplicación de software, un proceso de enfermería, hasta una pre-defensa de monografía o presentar un artículo científico.... Hoy 18 de septiembre del 2013, nuestra "alma mater" UNAN-Managua, se honra en celebrar exitosamente ese gran proceso de intercambio estudiantil, como lo es la XVI JUDC 2013 de FAREM Estelí. 


\section{La JUDC: su Naturaleza y sus Objetivos}

La JUDC se concibe como un evento académico/científico estudiantil, generado de la actividad investigativa durante el proceso de enseñanza-aprendizaje, bajo la conducción de un docente, como tutor, quien velará por transmitir la metodología de los procesos de investigación, innovación, emprendimiento, y elaboración de proyectos, con miras a consolidar la formación de los futuros profesionales.

La Jornada Universitaria de Desarrollo Científico (JUDC) es el espacio institucional coordinado por la Comisión de Investigación de cada Facultad de la UNAN-Managua, la que se encuentra conformada por delegados facultativos, centros de investigación, el POLISAL y un miembro de ATD.

\section{Objetivos de la JUDC}

\section{Son objetivos de la Jornada Universitaria de Desarrollo Científico los siguientes:}

- Promover la participación de los estudiantes universitarios, en actividades de investigación, innovación, emprendimiento y desarrollo de la propiedad intelectual con la finalidad de elevar sus niveles científicos y académicos.

- Crear en el estudiante universitario un genuino interés por el quehacer científico y técnico, a fin de contribuir a su desarrollo integral como futuro profesional.

- Vincular a la comunidad universitaria con la realidad nacional e internacional, a través del estudio de problemáticas y fenómenos en cada una de las áreas del conocimiento que presente interés para los estudiantes.

- Divulgar a la comunidad académica y científica la importancia de la Jornada Universitaria de Desarrollo Científico, como evento que promueve el quehacer docente e investigativo de la Universidad.

- Presentar a los estudiantes investigadores y docentes que se desenvuelven como tutores y/o jurados, un documento que contiene guías estandarizadas y consensuadas sobre los diferentes instrumentos de evaluación que se utilizan en los procesos de investigación y los aspectos normativos de la JUDC.

- Contribuir al desarrollo económico, político, social y ambiental del país.

\section{La XVI JUDC 2013 FAREM-Estelí, UNAN-Managua}

En esta XVI JUDC 2013 de FAREM Estelí, van a presentarse 129 trabajos, lo cuales están organizados en 12 diferentes Mesas de trabajos, distribuidos de la siguiente forma:

\begin{tabular}{|l|l|c|c|c|l|l|}
\hline $\mathbf{N}^{\circ}$ & \multicolumn{1}{|c|}{ Nombre mesa } & $\begin{array}{c}\mathbf{N}^{\circ} \text { de } \\
\text { trabajos }\end{array}$ & $\begin{array}{c}\mathbf{N}^{\circ} \text { de } \\
\text { estudiantes }\end{array}$ & $\begin{array}{c}\mathbf{N}^{\circ} \text { de } \\
\text { tutores }\end{array}$ & \multicolumn{1}{|c|}{ Fechas } & Lugar \\
\hline 1 & $\begin{array}{l}\text { Contaduría Pública y } \\
\text { finanzas }\end{array}$ & 24 & 57 & 6 & $\begin{array}{l}\text { Miércoles y jueves } \\
\text { (noche). Sábado }\end{array}$ & $\begin{array}{l}602,603 \text { y } \\
301 \text { (Sábado) }\end{array}$ \\
\hline
\end{tabular}




\begin{tabular}{|l|l|c|c|c|l|l|}
\hline 2 & Mercadotecnia & 22 & 26 & 1 & $\begin{array}{l}\text { Miércoles y jueves } \\
\text { (noche) }\end{array}$ & $\begin{array}{l}\text { Aula } 504 \text { y } \\
505\end{array}$ \\
\hline 3 & $\begin{array}{l}\text { Administración de } \\
\text { Empresas }\end{array}$ & 14 & 39 & 2 & $\begin{array}{l}\text { Miércoles y jueves } \\
\text { (noche) y jueves } \\
\text { (Mañana) }\end{array}$ & $\begin{array}{l}\text { Sala } 2 \\
\text { biblioteca }\end{array}$ \\
\hline 4 & Economía & 8 & 21 & 1 & Jueves (tarde) & Auditorio \\
\hline 5 & Ingeniería & 8 & 31 & 3 & Miércoles (tarde) & Auditorio \\
\hline 6 & Turismo sostenible & 7 & 26 & 3 & Jueves (Mañana) & $\begin{array}{l}\text { Sala } 1 \\
\text { Biblioteca }\end{array}$ \\
\hline 7 & $\begin{array}{l}\text { Ciencias Ambientales y } \\
\text { Biología }\end{array}$ & 7 & 18 & 5 & Sábado & Aula 611 \\
\hline 8 & Física-Matemática & 6 & 12 & 2 & Sábado & Aula 609 \\
\hline 9 & $\begin{array}{l}\text { Trabajo social (Proto- } \\
\text { colos) }\end{array}$ & 4 & 11 & 1 & Jueves (noche) & Aula 606 \\
\hline 10 & $\begin{array}{l}\text { Ciencias de la } \\
\text { educación }\end{array}$ & 7 & 13 & 4 & Sábado & $\begin{array}{l}\text { Sala de Post- } \\
\text { grado }\end{array}$ \\
\hline 11 & $\begin{array}{l}\text { Ensayos sobre la } \\
\text { problemática social }\end{array}$ & 9 & 22 & 3 & $\begin{array}{l}\text { Miércoles y jueves } \\
\text { (noche) }\end{array}$ & $\begin{array}{l}\text { Sala de Post- } \\
\text { grado }\end{array}$ \\
\hline 12 & Foto-Etnografía** & 13 & 32 & 1 & Viernes (tarde) & $\begin{array}{l}\text { Sala de Post- } \\
\text { grado }\end{array}$ \\
\hline
\end{tabular}

** Estas mesas temáticas se están abriendo por primera vez en esta XVI JUDC de la FAREMEstelí

Luego de una propuesta técnica realizada por una "Comisión Ad-Hoc", se procedió a discutir cada una de las guías de evaluación, y en pleno de la Comisión de Investigación de UNAN-Managua, se corrigió y aprobó las “Nuevas Guías de Evaluación de la Normativa de la JUDC 2012”, según el Acta Número 07 / 08-2012 del 24 y 31 de Julio 2012.

Esta XXXII JUDC-2013 de UNAN-Managua y XVI JUDC 2013-FAREM-Estelí, está organizada con esta nueva Normativa de JUDC, que contiene diez "Nuevas Guías de Evaluación de la JUDC 2012".

Así mismo, se incluye en la nueva normativa, las normas generales para la redacción del resumen de los trabajos de investigación científica, adaptado de la Dra. Barbara Gastel, MD, MPH., y las Normativas APA para citas y referencias bibliográficas en trabajos académicos, adaptado por la MSc. Anielka Carballo. Esta nueva normativa incluye cuatro nuevos tipos de trabajos a presentar a partir del año pasado, tales son: a) Procesos de Enfermería, b) Planes de Negocios, c) Aplicaciones Educativas, Proyectos Educativos Basados en Tecnologías, d) Artículos Científicos.

\section{Los Avances de la XVI JUDC 2013-FAREM-Estelí, UNAN-Managua}

Los avances de la XVI JUDC-2013 de la FAREM Estelí, pueden valorarse a la luz de los siguientes indicadores del cumplimiento de la misma, en relación a la JUDC realizada en el año 2011 y 2012. 
- XIV Jornada Universitaria de Desarrollo Científico (JUDC)-2011 En el año 2011, se presentaron 65 Trabajos, con 190 Estudiantes participantes y 27 tutores.

- XV Jornada Universitaria de Desarrollo Científico (JUDC)-2012 En el año 2012, se presentaron 102 Trabajos (+36\%), con 313 Estudiantes participantes (+40\%) y 34 tutores $(+21 \%) \ldots$. Todo esto organizado en 12 salas.

- XVI Jornada Universitaria de Desarrollo Científico (JUDC)-2013 Ahora en este año 2013, se van a presentaron 129 Trabajos (+21\%), con 308 Estudiantes participantes $(-1,50 \%)$ y 32 tutores $(-5,90 \%) \ldots$. Todo esto organizado en las mismas 12 salas.

\begin{tabular}{|c|c|c|c|c|c|c|}
\hline \multirow{2}{*}{ Facultades } & \multicolumn{2}{|c|}{ Trabajos presentados } & \multicolumn{2}{c|}{ Estudiantes participantes } & \multicolumn{2}{c|}{ Tutores } \\
\cline { 2 - 7 } & $\mathbf{2 0 1 1}$ & $\mathbf{2 0 1 2}$ & $\mathbf{2 0 1 1}$ & $\mathbf{2 0 1 2}$ & $\mathbf{2 0 1 1}$ & $\mathbf{2 0 1 2}$ \\
\hline FAREM-Estelí & 65 & $\begin{array}{c}102 \\
(+36 \%)\end{array}$ & 190 & $\begin{array}{c}313 \\
(+40 \%)\end{array}$ & 27 & $\begin{array}{c}34 \\
(+21 \%)\end{array}$ \\
\hline
\end{tabular}

La XVI JUDC-2013 de la FAREM Estelí, está organizada de manera más efectiva y eficiente, ya que se tiene planificado que se van a presentar 129 Trabajos, se tiene una participación esperada de 308 estudiantes y participación de 32 tutores. Estas cifras muestran un gran Avances de la XVI JUDC 2013 de FAREM-Estelí, en comparación con la JUDC del año 2011 y 2012.

La XVI JUDC-2013 de la FAREM Estelí, está organizada en conjunto con la unidad administrativa de esta Facultad Multidisciplinaria, se tiene un presupuesto para esta JUDC 2013, por un monto total igual a 113,100,00 C\$, (ciento trece mil, cien córdobas netos). Esto significa que estamos superando en eficiencia y efectividad al menos en un $21 \%$, en comparación con el presupuesto de la JUDC del año 2012. La inversión unitaria por cada trabajo presentado en la JUDC 2013, es de 1037.37 C\$. Cabe destacar que este presupuesto estuvo disponible a tiempo en la administración de FAREM Estelí, desde antes de comenzar esta XVI JUDC facultativa.

Otros Avances de la FAREM-Estelí, que fortalecen esta XVI JUDC 2013, son:

1. La Revista Científica de FAREM Estelí desarrollada en plataforma digital "M.A. Tecnología y Desarrollo Humano". FAREM ESTELI que fue la primera facultad el lograrlo, iniciando en marzo del 2012 y ya cuenta con 6 números

2. Las Líneas de Investigación APROBADAS EN 2010 POR EL CONSEJO FACULTATIVO DE FAREM ESTELI, que fue la primera facultad el lograrlo.

El Análisis de frecuencias del número de líneas de investigación de cada facultad/Centro o Instituto de Investigación de UNAN-Managua, permite destacar que éstas responden a las prioridades del PNDH en el siguiente orden de importancia:

- Facultad de Ciencias e Ingenieras, con 79 líneas de investigación funcionales, que representan el $24.2 \%$.

- Facultad de Humanidades y Ciencias Jurídicas, con 53 líneas de investigación, que representan el $16.2 \%$.

- La FAREM Matagalpa, con 32 líneas de investigación, que representan el 9.8\%. 
- Facultad de Ciencias Médicas, con 28 líneas de investigación, que representan el 8.6\%.

- $\quad$ FAREM Estelí, con 25 líneas de investigación, que representan el $7.6 \%$.

- La FAREM Chontales, con 22 líneas de investigación, que representan el $6.7 \%$.

- CIRA, con 18 líneas de investigación, que representan el 5.5\%.

Estas cifras demuestran claramente los Avances en la FAREM Estelí, se destaca aquí los Avances relevantes con esta XVI JUDC. También, hay avances importantes, tanto en Publicaciones científicas, como en la Organización de las Líneas de Investigación por Departamento docente, como en su Aporte Estratégico en apoyo al PNDH.

"Vivimos en una época de cambios, que están cambiando la ápoca en que vivimos. Tal como se destaca en el lema de esta XVI JUDC, "Por la Excelencia Académica, Investigando e Innovando", esto significa que vivimos "tiempos de compromiso universitario, tiempos de aportar al desarrollo humano de las y los nicaragüenses" y ese es el compromiso que dejamos en esta XVI JUDC.

En una realidad tan dinámica como la que se vive actualmente en nuestra comunidad universitaria, nuestros estudiantes universitarios y los profesionales nicaragüense de las diferentes áreas de la ciencia, debemos sentirnos altamente estimulados con la realización de este XVI JUDC, FAREM Estelí, porque responde a la necesidad de modernización de estudiantes y profesionales de las diferentes áreas del conocimiento. Esta XVI JUDC, FAREM Estelí, destaca el Modelo Educativo De Nuestra Alma Mater, centrado en nuestros estudiantes, centrado en nuestra gente, un modelo educativo que promueve el aprendizaje autónomo y colaborativo, que nos conduce de la mano para reconocer la importancia de la visión multidimensional de la investigación científica, lo cual se sintetiza en el Modelo I-D-i, de UNAN-Mga, declarado explícitamente desde 2012, modelo que conlleva a la aplicación de dos grandes enfoques: el enfoque antropocéntrico y el enfoque sistémico, lo cual implica la integración real de la investigación, la innovación y el emprededurismo.

Este largo camino se inicia desde la investigación observacional, con enfoque cualitativo, así como la integración sistémica con la investigación observacional con enfoque cuantitativo, transitando por el camino de reconocer y diferenciar las diversas aplicaciones de los enfoques multi, inter y transdisciplinarios de la investigación científica. Este transito llega hasta completarse con las investigaciones estratégicas y aplicadas de carácter experimental. Todo el1o, destaca la aplicación de diversos métodos y enfoques de investigación, los que en su conjunto contribuyen a resolver problemas reales del mundo en que vivimos. Este es un ejemplo de la integración del Modelo Educativo y el Modelo I-D-i, el cual se facilita directamente desde la JUDC

En resumen, esta XVI JUDC, FAREM Estelí, organizada con 12 salas que permitirán 129 ponencias, con 308 Estudiantes participantes y 32 tutores, más la conferencia magistral del Cro. Eduardo López, Prof. Titular de FAREM Estelí y Representante del cuerpo docente ante el Consejo facultativo de FAREM Estelí, es un excelente espacio para divulgar el nuestro que hacer de investigación de FAREM Estelí, y compartir experiencias exitosas de científico-metodológicas relacionadas con los temas que se abordan, para aportar en dar respuesta a los problemas que 
demanda la sociedad nicaragüense, en correspondencia con el "Plan Nacional de Desarrollo Humano, 2012-2016", y por esta vía ir mejorando la productividad científica del país, en aras de mejorar el nivel y calidad de vida de la familia nicaragüense.

Por tales razones, felicito de manera muy especial a las autoridades facultativas a la Cra. Decana Reyna Sevilla Midence, al Cro. Vicedecano Alejandro Raudez, a las Cras. y Cros. del Consejo Facultativo de FAREM Estelí, así también felicito a la Comisión de Investigación de FAREM Estelí, organizadora de este XVI JUDC, y en particular a la Cra. Beverly Castillo Herrera, les felicito de todo corazón por la calidad y pertinencia del contenido de esta JUDC, que desde ya se asegura la aplicabilidad de los conocimientos que se compartirán durante los cuatro días de esta JUDC, tanto en la docencia universitaria, como en investigaciones en el campo profesional.

Muchas felicidades y muchos éxitos en esta XVI JUDC, FAREM Estelí. 